\title{
Are we going to Alpha Centauri?
}

\section{Didier Queloz, Mejd Alsari*}

\begin{abstract}
Nobel Prize Laureate Didier Queloz talks about realistic ways to explore Proxima Centauri b and other potentially habitable planetary systems such as TRAPPIST-1 using technologies that are currently available. He also discusses his interdisciplinary research activities on abiogenesis and the search for life on other planets. See video at https://youtu.be/RODr30duRrg.
\end{abstract}

Mejd Alsari (MA). Does the Drake Equation ${ }^{1,2}$ define a sort of roadmap for astronomers in their quest for understanding our universe when you read it from left to right?

Note: the Drake Equation predicts the number of extant advanced technical civilizations possessing both the interest and the capability for interstellar communication as $N=R^{*} \times f_{\mathrm{p}} \times n_{\mathrm{e}} \times f_{1} \times f_{\mathrm{i}} \times f_{\mathrm{c}} \times L$, where: ${ }^{1}$

- $R^{\star}$ is the mean rate of star formation averaged over the lifetime of our Galaxy;

- $f_{\mathrm{p}}$ is the fraction of stars with planetary systems;

- $n_{\mathrm{e}}$ is the mean number of planets in each planetary system with environments favourable for the origin of life;

- $f_{1}$ is the fraction of such favourable planets on which life develops;

- $f_{\mathrm{i}}$ is the fraction of such inhabited planets on which intelligent life arises during the lifetime of the local star;

- $f$ is the fraction of planets populated by intelligent beings on which an advanced technical civilization arises during the lifetime of the local star;

- $L$ is the lifetime of the technical civilization.

Didier Queloz (DQ). No. I think the Drake Equation is based on a very simplistic concept of life; that the only possible life would be like the one on Earth. The problem with that equation is that it is only relevant for the Search for Extra-Terrestrial Intelligence (SETI) experiment, which looks at intelligent life as it is today on Earth on a global statistical way. ${ }^{2-4}$ If you look at the history of life on Earth, it was invisible for most of the time. I think the Drake Equation is targeting the wrong public. It has given a very nice simplification to build up a program like SETI, looking for radio signals. ${ }^{5,6}$ Right now, this is not the way people are addressing the problem of extra-terrestrial life.

The problem is clearly not about the number of stars, the number of planets, etc. We know this. There are planets everywhere. The question that should be asked here is 'What defines a planet that can sustain life?'?

There are two ways to do that. One can be lacking creativity and use the Drake Equation, which practically relates to Earth. Or one can try to be creative and say 'There are many planets different from Earth that could produce life, which might be different from the one we are familiar with'. It might be different but it's still chemistry, different kind of chemistry. Maybe life is not visible, almost invisible. Maybe there is life on Venus, ${ }^{8}$ maybe there is life on Mars. ${ }^{9}$ We are not very good at finding that right now. But maybe there is life there and we are not looking at the right stuff.

Given the diversity of the planetary systems we've discovered, I think it's safe to hypothesize an extremely high diversity in the chemistry of these planets. Whether such a diversity in chemistry can lead to life, that's open to debate. It's something that can be

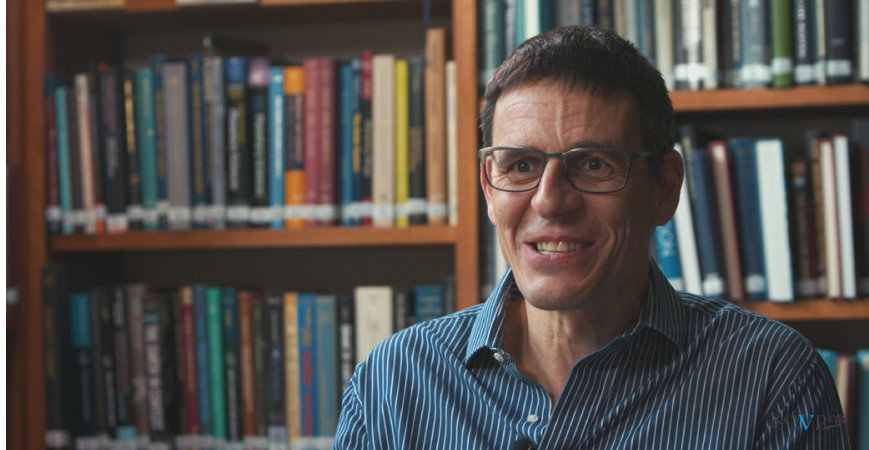

Figure 1 | Prof. Didier Queloz, Professor of Physics, Cavendish Laboratory University of Cambridge.

tested. It's a chemistry experiment that needs to be done to address these fundamental questions. What is the origin of the chemistry of life on Earth? How far can we divert from this chemistry on Earth to make another chemistry that leads to something that looks like life? ${ }^{10-12}$

Therefore, I think that from this perspective the Drake Equation does not tell you anything at all.

MA. What do you think of the SETI program? ? $^{5,6}$

DQ. The SETI program is targeted to a very specific question: 'Could you find a civilization on the base of the signals they produce?'

SETI has been focussing mostly on radio signals, but there could also be light signals. I think there are no limits in science. If people believe this is what they want to look for, I am very happy for them to do that. The problem I have with the SETI program is that I would never do that because I think it's an extremely frustrating program, in which you learn nothing from a null result. When you do an experiment you really have to acknowledge a null result. The null result should tell you as much as the result.

To me it's a quest that is looking at something that you have an idea of. I think it's a beautiful quest because I can understand the myth behind it, I can understand the impact. But if you find nothing you are still in the same situation.

In the field of exoplanets you can look for a planet, you can detect a planet, and ask questions about the origin of life in this planet. You measure the chemistry of this planet, you understand something about the chemistry, the geochemistry, the cycle of the chemistry, and possible imbalances in the chemistry of the atmosphere. Whether there is life or not, you can understand if there are the minimum conditions on these planets to support life. Then you can start elaborating on the likelihood of life developing on these planets.

The other problem I have with SETI is that, let's imagine there is 
a civilization of fish. I don't know how you can talk to fish. I think people working at SETI should first try with, for example, jellyfish. They could take a couple of jelly fish and try to exchange information with them here on Earth.

That's exactly the kind of problem they are facing. I understand that SETI is a vision of the sixties. ${ }^{5}$ I love it because it's an easy try. It had to be tried. It's easy. You can point to the stars with a radio telescope, and if you detect something you would know if that comes from an intelligent life.

People have been looking at lots of stars right now with the SETI program. Whether this program should continue or not, again, it depends on what they are really looking for. But then, at the end, if they inspect one billion stars and they get nothing, what did they learn about life in the universe? Nothing. They just learn that there is no intelligent life broadcasting signals outside Earth at the time they made the measurement. This does not mean that there wasn't life in the remote past or that there will be life in the remote future on that planet.

These are the problems I have with SETI right now. But if there are people working on this, I am always curious to hear what they are doing. This is part of science, the diversity of science.

MA. Can you tell us about the work you've done in collaboration with a group at the Laboratory of Molecular Biology (LMB) here in Cambridge, where you talk about the origin of RNA precursors in exoplanets? ${ }^{\text {13 }}$

DQ. Right now we are trying to define the way to make progress first with the origin of life on Earth and then with the possibility of life on other planets. There are lots of discussions on what is the best way to move forward. There are a couple of elements we all agree on. When you look for the origin of life (abiogenesis) on Earth, there is a limit in what you can get because we have no memory of the past on Earth. There is no way to know exactly how life emerged back then, except to look at what is the current structure of life.

The current mechanism of life is the result of an extremely complex evolution. Consider for example the first and last versions of the steam engine. There has been so much progress. In a similar way, life has optimised itself in the way we see it today. There may be some elements related to the primordial life in the current living mechanisms, but these are hard to detect.

In the work you mentioned, we decided to go as back as we could. We started with the fact that there is a limit in the number of amino acids that life is using. We asked 'Can we start from that to define what the conditions for life are?'14,15

That's kind of going to the simplest possible assumption. It doesn't mean that when we have amino acids we will get life. Nobody really has been able to connect the dots yet. There are people working on that. I am optimistic that at some point in a lab, here on earth, people will be able to do that, i.e. try to assemble the amino acids in a way that they produce some kind of replication mechanism.

Then we look at this and we ask the question 'Could you do the chemistry, the science conditions, imagine a planet, and how does it work?' We found out that the light from a star could act as an energy trigger. So we looked at the possibility of life from the stars. We tried to measure that. We compared it with the chemistry. We did chemistry experiments by bringing 'astrophysics' light, in a way, not just a lamp, but light that is properly calibrated. ${ }^{15}$

These are all the kinds of connections we are trying to establish right now. That's the way the field is trying to evolve, which is trying to bring different pieces of knowledge together: the astrophysics knowledge from a planet, the atmosphere of a planet, the geophysics knowledge from Earth, and the Solar System. We also wanted to see how far we could learn about plate tectonics, volcanic activity, and magnetic fields on these exoplanets. This is part of the equation. Some people assume that you absolutely need plate tectonics to recycle carbon. ${ }^{16,17}$ If this is the only way to recycle carbon, I guess we need that, otherwise we would end up like Venus. Venus may have looked like Earth half a billion years ago. It was experiencing some plate tectonic recycling. If you imagine the first inhabitants of Earth looking at Venus, they would have seen a kind of blue planet as well. When plate tectonics stopped in Venus, ${ }^{18}$ carbon recycling stopped, leading to a runaway greenhouse effect. ${ }^{17}$

All these elements are very interesting and we can study them by combining all these fields. That's the approach we've started here. We are not the only ones. There are a couple of people in Harvard.

There is a global initiative, which is supported by the Simons Foundation right now. ${ }^{19}$ We are trying to connect all this together, which to me is extremely interesting. I'm trying to promote this idea and I hope that this Nobel Prize will help my voice to be heard that we are building a new field.

We are facing what's called red tape effect, a bureaucratic effect, which is if you are an astrophysicist and you want to ask for money to do a chemistry experiment, you can't. The astrophysics panel will tell you 'Well, this is astrophysics you should ask chemistry'. The chemistry panel will tell you 'You are an astrophysicist you shouldn't ask us'.

So there is no funding. This is the kind of challenge you face when you do science between different scientific topics, which are usually completely separated. ${ }^{20}$ We don't even have the same science council for astronomers and chemists.

How can we assemble a sensible critical mass of people that will be able to operate together and come up with a real roadmap? I don't think we have a clear roadmap right now but there are lots of elements, which are interesting. These are related to the investigation of the most basic elements of life as we know it, which is to find the conditions on other planets and then possibly explore deviations from that. If you don't have the same chemistry, could you trick a little bit the chemistry and still have something, which could be different amino acids? You might come up with something similar, maybe not exactly the same, which will work as well. You might end up with a different kind of life, which is based on a different functioning mechanism that at the end leads to the same kind of global life kind of mechanism.

I think this is the adventure we are going to embark upon. I am pretty optimistic that, given all the efforts, all the brains, all the tools we have, we should get closer and closer to an answer. It may take a hundred years still, which in terms of science is nothing.

So that's the kind of life roadmap that I would see while it is not yet clearly defined.

MA. In 2016 you discovered planets orbiting a star called TRAPPIST-1, what do we know about this system? ${ }^{21}$

Note: TRAPPIST is an acronym for TRAnsiting Planets and PlanetsImals Small Telescope-South. ${ }^{22,23}$

DQ. That's a great work that I did with Belgian colleagues and Michaël Gillon is the leader of this exercise. I think this is fascinating because we are trying to trick the system here. We are using smaller

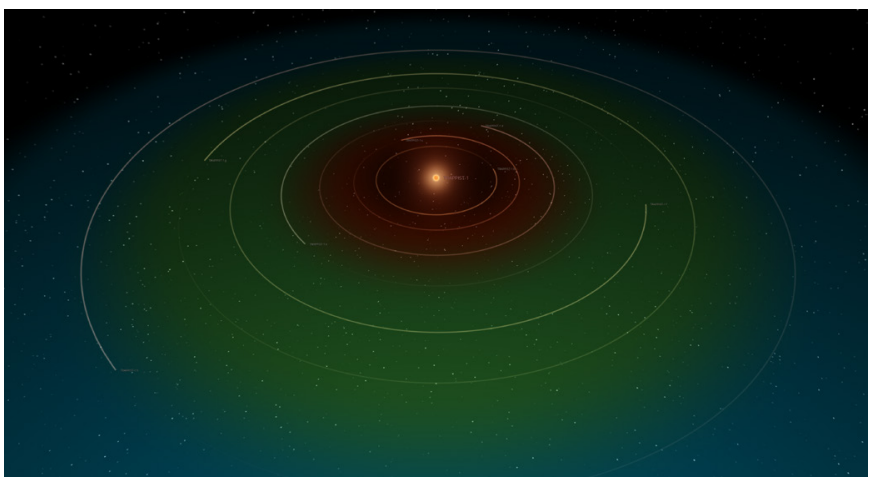

Figure 2 | TRAPPIST-1 system.(habitable zone in green colour). 
stars, cool stars, M stars, ${ }^{24,25}$ to compensate the fact that we haven't found a true Earth, but we have plenty of planets similar to Earth. The idea is that if you look for an Earth-sized planet very close to a star and you want that planet to be potentially habitable you need to make sure the planet is not roasted by the star. By looking for cooler stars, $\mathrm{M}$ stars, you have the right distance to have the same kind of flux coming from the star that we experience here on Earth.

We found the TRAPPIST-1 system and there are two or three planets, which are clearly like Earth in terms of mass and size. These planets are in a region that we call the habitable zone. It's a region where if you took Earth as it is and you moved it within this habitable zone it would maintain liquid water on its surface. ${ }^{16,21}$

The assumption is that within the habitable zone you would have atmosphere and geophysics similar to the one on Earth. We don't have such information for the TRAPPIST-1 system. But the fact that those planets are within the habitable zone gives some boundary conditions.

There is a real interest to go a little bit deeper about these planets and to try to detect their atmosphere. Low mass stars emit a different kind of radiation from the Sun. These stars are very active when they are young (superluminous pre-main-sequence phase). ${ }^{26,27}$ The Sun slowed down very quickly and then it became nice; although you would still have eruptions from time to time. M-stars are very different and took long time to cool down. There is a lot of activity, lots of X-rays. So we don't really like M stars because of this. They emit a lot of high energy particles. ${ }^{28,29}$

It is not very clear whether an atmosphere in a planet orbiting an M-star would survive. But let's find out. ${ }^{21}$ Assuming the atmosphere would survive, how would it look like? ${ }^{30}$

Here we are talking about another kind of star, with a very close planet. So nobody knows well about this. But again let's have a look. We have the James Webb Telescope (JWST) and maybe it could do something. ${ }^{30-32}$

The ambiguity of this program is that if the atmosphere is very thick like for example $200-500 \mathrm{~km}$ it will be detected by the JWST. However if the atmosphere is like the one on Earth, 10-20 km, we won't see anything. ${ }^{32}$ So we should be able to know whether there is an atmosphere, which doesn't look much like Earth, because we don't have $500 \mathrm{~km}$ atmosphere on Earth. Or we might detect no atmosphere, which doesn't tell that there is no atmosphere. It tells that we haven't detected anything, but we could still have a $10 \mathrm{~km}$ atmosphere or no atmosphere at all like on Mars.

TRAPPIST-1 is an interesting system because if you detect a thick atmosphere in any of its planets, you can almost reject life on it, but if you don't detect atmosphere there is still hope that there is life there. In this case you wait for the next generation of more sensitive instruments. So it's science building up on the past.

TRAPPIST- 1 is a fascinating system. There may be others. We are looking for other similar systems. Who knows what kind of surprises we will be able to find. I think it's great because for the first time we can really try something and we can at least get some data. It is not about talking and imagining something. We have a target, we have the tool, let's find out!

I think it's a fantastic adventure we are living right now.

MA. The closest potentially habitable exoplanet is Proxima Centauri $b$ that was discovered in $2016 .{ }^{33}$ If we decide to send a probe there, with our current propulsion systems, it would take some 50,000 years. So what are the most viable candidates for new propulsion systems that are currently under study? ${ }^{34-39}$

DQ. I don't really like the idea of sending a probe. I think it's a sci-fi dream. I think it's a misuse of funds right now. You can study a lot of stuff just by looking. I think it would be much better to design the kind of equipment necessary to retrieve as much data as we can before sending a probe. I think we are a hundred years behind sending any probe at that distance. If you send a probe with the current technology, in 10,000 years we might have a technology to go much

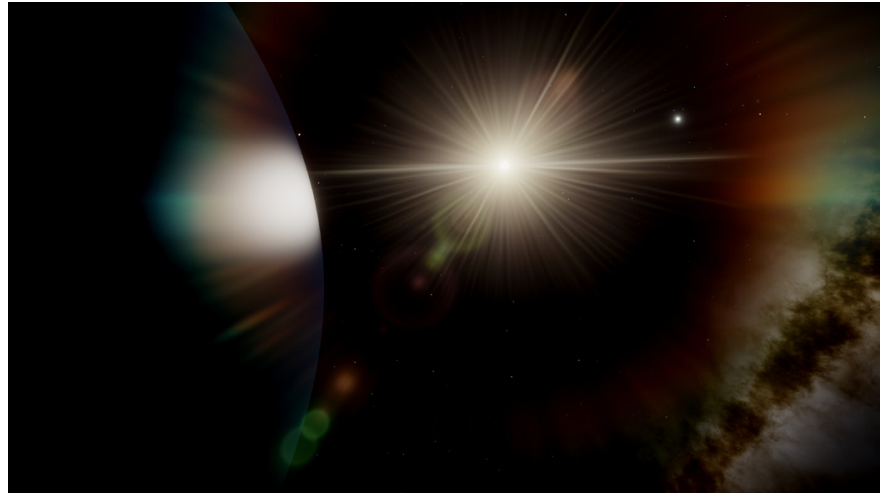

Figure 3 | Artist impression of Alpha Centauri system showing Proxima Centauri b, Proxima Centari, and Alpha Centauri A and B.

faster and we would still have this probe on its way when the new one passes it.

I know that there are people dreaming about this. There are private sponsors that would like to do that ${ }^{40,41}$ I personally regret that they are not talking to astronomers about this. I love the idea to look for life on Proxima Centauri b, it's a very good program. But sending a probe is not a good idea. We should build equipment to just look at it. With that we should be able to tell a lot already without physically going there.

Now, if you want to go there, the problem we have to face, is that you better go fast because otherwise it takes too long. The speed has to be quite significant. When you start reaching $1 \%$ of the speed of light the mass becomes bigger. ${ }^{42,43}$ Moreover how will you slow down the probe? If you just have a flyby of one second around the planet, what's the purpose?

I think we should try to engage better with the people that would like to have this program. I would really like to invite people interested in this topic, especially private sponsors, to talk to astronomers and try to come up with a realistic program.

This idea of sending a probe to Proxima Centuari, I think, is just a dream right now. We shouldn't spend money in this way. You can still dream, it's fine, but spending money on that is just a mistake to me.

\section{References}

1 C. Sagan. Direct contact among galactic civilizations by relativistic interstellar spaceflight. Planetary and Space Science 11, 485-498, (1963).

2 F. Drake \& D. Sobel. Is anyone out there? The scientific search for extraterrestrial intelligence. (1992).

3 J. Tarter. The Search for Extraterrestrial Intelligence (SETI). Annual Review of Astronomy and Astrophysics 39, 511-548, (2001).

4 SETI. SETI website, https://www.seti.org/, (2020).

5 G. Cocconi \& P. Morrison. Searching for Interstellar Communications. Nature 184, 844-846, (1959).

6 SETI. Early SETI: Project Ozma, Arecibo Message, https://www.seti.org/seti-institute/project/details/early-seti-project-ozma-arecibo-message, (2020).

7 J. L. Grenfell. A review of exoplanetary biosignatures. Physics Reports 713, 1-17, (2017).

8 S. S. Limaye, R. Mogul, D. J. Smith, A. H. Ansari, G. P. Słowik \& P. Vaishampayan. Venus' Spectral Signatures and the Potential for Life in the Clouds. Astrobiology 18, 1181-1198, (2018)

9 F. Westall, F. Foucher, N. Bost, M. Bertrand, D. Loizeau, J. L. Vago, G. Kminek, F. Gaboyer, K. A. Campbell, J.-G. Bréhéret, P. Gautret \& C. S. Cockell. Biosignatures on Mars: What, Where, and How? Implications for the Search for Martian Life. Astrobiology 15, 998-1029, (2015)

10 H. J. Cleaves, C. Butch, P. B. Burger, J. Goodwin \& M. Meringer. One Among Millions: The Chemical Space of Nucleic Acid-Like Molecules. Journal of Chemical Information and Modeling 59, 4266-4277, (2019). 
11 V. B. Pinheiro, A. I. Taylor, C. Cozens, M. Abramov, M. Renders, S. Zhang, J. C. Chaput, J. Wengel, S.-Y. Peak-Chew, S. H. McLaughlin, P. Herdewijn \& P. Holliger. Synthetic Genetic Polymers Capable of Heredity and Evolution. Science 336, 341, (2012).

12 S. Hoshika, N. A. Leal, M.-J. Kim, M.-S. Kim, N. B. Karalkar, H.-J. Kim, A. M. Bates, N. E. Watkins, H. A. SantaLucia, A. J. Meyer, S. DasGupta, J. A. Piccirilli, A. D. Ellington, J. SantaLucia, M. M. Georgiadis \& S. A. Benner. Hachimoji DNA and RNA: A genetic system with eight building blocks. Science 363, 884, (2019).

13 P. B. Rimmer, J. Xu, S. J. Thompson, E. Gillen, J. D. Sutherland \& D. Queloz. The origin of RNA precursors on exoplanets. Science Advances 4, eaar3302, (2018).

14 J. Xu, D. J. Ritson, S. Ranjan, Z. R. Todd, D. D. Sasselov \& J. D. Sutherland. Photochemical reductive homologation of hydrogen cyanide using sulfite and ferrocyanide. Chemical Communications 54, 5566-5569, (2018).

15 B. H. Patel, C. Percivalle, D. J. Ritson, C. D. Duffy \& J. D. Sutherland. Common origins of RNA, protein and lipid precursors in a cyanosulfidic protometabolism. Nature Chemistry 7, 301-307, (2015).

16 J. F. Kasting, D. P. Whitmire \& R. T. Reynolds. Habitable Zones around Main Sequence Stars. Icarus 101, 108-128, (1993).

17 R. K. Kopparapu, R. M. Ramirez, J. SchottelKotte, J. F. Kasting, S. Domagal-Goldman \& V. Eymet. HABITABLE ZONES AROUND MAIN-SEQUENCE STARS: DEPENDENCE ON PLANETARY MASS. The Astrophysical Journal 787, L29, (2014).

18 T. K. P. Gregg. in Treatise on Geophysics (Second Edition) (ed Gerald Schubert) 307-325 (Elsevier, 2015).

19 S. Foundation. SCOL Project: Search for Habitable Planets Eclipsing Ultra-Cool Stars, https://www.simonsfoundation.org/team/didier-queloz/, (2020).

20 E. Bozhkova. Interdisciplinary proposals struggle to get funded, https://www.nature.com/news/interdisciplinary-proposals-struggle-to-get-funded-1.20189, (2016)

21 M. Gillon, E. Jehin, S. M. Lederer, L. Delrez, J. de Wit, A. Burdanov, V. Van Grootel, A. J. Burgasser, A. H. M. J. Triaud, C. Opitom, B.-O. Demory, D. K. Sahu, D. Bardalez Gagliuffi, P. Magain \& D. Queloz. Temperate Earth-sized planets transiting a nearby ultracool dwarf star. Nature 533, 221-224, (2016). M. Gillon, E. Jehin, P. Magain, V. Chantry, D. Hutsemékers, J. Manfroid, D. Queloz \& S. Udry. TRAPPIST: a robotic telescope dedicated to the study of planetary systems. EPJ Web of Conferences 11, 06002, (2011).

23 M. Gillon, E. Jehin, A. Fumel, P. Magain \& D. Queloz. TRAPPIST-UCDTS: A prototype search for habitable planets transiting ultra-cool stars. EPJ Web of Conferences 47, 03001, (2013).

24 J. Liebert \& John E. Gizis. RIPhotometry of 2MASS-selected Late M and L Dwarfs. Publications of the Astronomical Society of the Pacific 118, 659-670, (2006).

25 Todd J. Henry, W. C. Jao, John P. Subasavage, Thomas D. Beaulieu, Philip A. Ianna, E. Costa \& René A. Méndez. The Solar Neighborhood. XVII. Parallax Results from the CTIOPI 0.9 m Program: 20 New Members of the RECONS 10 Parsec Sample. The Astronomical Journal 132, 2360-2371, (2006).

Luger \& R. Barnes. Extreme Water Loss and Abiotic O2 Buildup on Planets Throughout the Habitable Zones of M Dwarfs. Astrobiology 15, 119-143, (2015).

27 V. S. Meadows, G. N. Arney, E. W. Schwieterman, J. Lustig-Yaeger, A. P. Lincowski, T. Robinson, S. D. Domagal-Goldman, R. Deitrick, R. K. Barnes, D. P. Fleming, R. Luger, P. E. Driscoll, T. R. Quinn \& D. Crisp. The Habitability of Proxima Centauri b: Environmental States and Observational Discriminants. Astrobiology 18, 133-189, (2018)

28 B. Stelzer, A. Marino, G. Micela, J. López-Santiago \& C. Liefke. The UV and X-ray activity of the $\mathrm{M}$ dwarfs within $10 \mathrm{pc}$ of the Sun. Monthly Notices of the Royal Astronomical Society 431, 2063-2079, (2013).

29 P. J. Wheatley, T. Louden, V. Bourrier, D. Ehrenreich \& M. Gillon. Strong XUV irradiation of the Earth-sized exoplanets orbiting the ultracool dwarf TRAPPIST-1. Monthly Notices of the Royal Astronomical Society: Letters $\mathbf{4 6 5}$ L74-L78, (2016).

30 J. Lustig-Yaeger, V. S. Meadows \& A. P. Lincowski. The Detectability and Characterization of the TRAPPIST-1 Exoplanet Atmospheres with JWST. The Astronomical Journal 158, 27, (2019).
31 C. V. Morley, L. Kreidberg, Z. Rustamkulov, T. Robinson \& J. J. Fortney. Observing the Atmospheres of Known Temperate Earth-sized Planets withJWST. The Astrophysical Journal 850, 121, (2017).

32 J. Kalirai. Scientific discovery with the James Webb Space Telescope. Contemporary Physics 59, 251-290, (2018)

33 G. Anglada-Escudé, P. J. Amado, J. Barnes, Z. M. Berdiñas, R. P. Butler, G. A. L. Coleman, I. de la Cueva, S. Dreizler, M. Endl, B. Giesers, S. V. Jeffers, J. S. Jenkins, H. R. A. Jones, M. Kiraga, M. Kürster, M. J. López-González, C. J. Marvin, N. Morales, J. Morin, R. P. Nelson et al. A terrestrial planet candidate in a temperate orbit around Proxima Centauri. Nature 536, 437-440, (2016).

34 H. She, W. Hettel \& P. Lubin. Directed energy interception of satellites. Advances in Space Research 63, 3795-3815, (2019).

35 G. Hughes, P. Lubin, J. Griswold, B. Cook, D. Bozzini, H. O’Neill, P. Meinhold, J. Suen, J. Bible, J. Riley, I. Johansson, M. Pryor \& M. Kangas. Optical modeling for a laser phased-array directed energy system. Vol. 9226 OPO (SPIE, 2014).

36 T. Brashears, P. Lubin, G. Hughes, K. McDonough, S. Arias, A. Lang, C. Motta, P. Meinhold, P. Batliner, J. Griswold, Q. Zhang, Y. Alnawakhtha, K. Prater, J. Madajian, O. Sturman, J. Gergieva, A. Gilkes \& B. Silverstein. Directed energy interstellar propulsion of wafersats. Vol. 9616 OPO (SPIE, 2015).

37 R. Weed. Radioisotope Positron Propulsion, nasa.gov/directorates/spacetech/ niac/2018_Phase_I_Phase_II/Radioisotope_Positron_Propulsion, (2018).

38 P. Lubin. A Roadmap to Interstellar Flight, https://arxiv.org/abs/1604.01356, (2016).

39 N. Kulkarni, P. Lubin \& Q. Zhang. Relativistic Spacecraft Propelled by Directed Energy. The Astronomical Journal 155, 155, (2018).

40 T. Malik. Stephen Hawking Helps Launch Project 'Starshot' for Interstellar Space Exploration, https://www.space.com/32546-interstellar-spaceflight-stephen-hawking-project-starshot.html, (2016).

41 B. Initiatives. Breakthrough Initiatives, https://breakthroughinitiatives.org/, (2020).

42 A. Einstein. Zur Elektrodynamik bewegter Körper. Annalen der Physik 322, 891-921, (1905).

43 A. Einstein. Ist die Trägheit eines Körpers von seinem Energieinhalt abhängig? Annalen der Physik 323, 639-641, (1905).

\section{Conflicts of Interest}

There are no conflicts to declare.

\section{Acknowledgements}

M.A. acknowledges support from the President of the UAE's Distinguished Student Scholarship Program (DSS), granted by the UAE's Ministry of Presidential Affairs. Scientific Video Protocols thanks Dr Subhajit Sarkar and Dr Francesca Faedi for reviewing the manuscript.

\section{Author Contributions}

D.Q. was interviewed by M.A. M.A. wrote the manuscript.

\section{Keywords}

Exoplanets, abiogenesis, habitability.

\section{Article Information}

This article is licensed by Queloz et al. under a Creative Commons Attribution 4.0 International License (CC BY 4.0). 